\title{
VISIÓN BINOCULAR TRAS CIRUGÍA REFRACTIVA EN PACIENTES HIPERMÉTROPES
}

\section{BINOCULAR VISION FOLLOWING REFRACTIVE SURGERY IN HYPEROPIC PATIENTS}

\author{
GÓMEZ-DE-LIAÑO-SÁNCHEZ R ${ }^{1}$, PIEDRAHITA-ALONSO E ${ }^{2}$, ARIAS-PUENTE A ${ }^{1}$
}

\begin{abstract}
RESUMEN
Objetivo: Analizar si existen modificaciones de la visión binocular y motilidad ocular en pacientes hipermétropes operados de cirugía refractiva.

Métodos: Estudio prospectivo de 31 pacientes consecutivos operados de cirugía refractiva bilateral de hipermetropía entre mayo de 1999 y febrero de 2002. El protocolo de exploración incluyó la agudeza visual corregida, el error refractivo bajo cicloplejía, la motilidad ocular, la fusión en visión lejana, la estereopsis y el grado de amplitud de fusión, realizándose de nuevo al mes, 3 meses, 6 meses, un año y en sucesivas revisiones anuales.

Resultados: Tras la cirugía refractiva se han encontrado alteraciones de la agudeza visual, defectos hipermetrópicos residuales, así como cambios de anisometropía, que han influido en la situación oculomotora. El tipo de alteración sensorial más frecuentemente modificado ha sido la supresión de lejos, alterándose la estereopsis en mucho menor grado. Desde el punto de vista de motilidad ocular, en los pacientes estrábicos se ha encontrado una tendencia significativa en el sentido endotrópico ( $p=0,003)$. En los sujetos no estrábicos, aunque se han hallado modificaciones sensoriomotoras, han
\end{abstract}

\begin{abstract}
Purpose: To evaluate the changes in binocular vision and ocular motility in hyperopic patients undergoing refractive surgery.

Methods: Prospective study of 31 consecutive patients who underwent bilateral refractive surgery for hyperopia between May 1999 and February 2002. The examination included best-corrected visual acuity, cycloplegic refraction, ocular motility, fusion at distance, stereopsis and fusional amplitudes, before and at one month, 3 months, 6 months, one year after surgery and annually thereafter.

Results: After refractive surgery, there were changes in visual acuity, residual hyperopic refractive errors, and anisometropic changes that influenced the oculomotor status. The most frequent sensory modifications were suppression at distance, with much less change in stereopsis. From the point of view of ocular motility, we found a significant tendency to esotropia in strabismic patients $(\mathrm{p}=0.003)$. In non-strabismic patients, we also found sensorimotor modifications, but of less intensity and with fewer consequences than in strabismic patients. Sensorial and/or motor decompensation appeared in
\end{abstract}

Recibido: 8/6/05. Aceptado: 20/2/06.

Centro Oftalmológico Gómez de Liaño. Madrid. España.

1 Doctor en Medicina.

2 Diplomado en Óptica y Optometría.

Comunicación presentada parcialmente en el LXXVIII Congreso de la S.E.O. (Murcia 2002).

Los autores no tienen interés comercial ni han recibido apoyo económico en la investigación en la que se basa este artículo.

Correspondencia:

Rosario Gómez de Liaño

$\mathrm{C} /$. Fortuny, $51,4^{\circ} \mathrm{C}$

28010 Madrid

España

E-mail: rgomezdeliano@med.ucm.es 
sido de menor intensidad y repercusión que en los sujetos estrábicos. Un $25 \%$ de los pacientes estrábicos presentó una descompensación sensorial y/o motora, y un $28,57 \%$ del mismo grupo mostró síntomas astenópicos.

Conclusiones: En los pacientes con visión binocular normal existieron leves modificaciones binoculares que no tuvieron repercusión sintomática. Sin embargo, en los pacientes estrábicos se modificó la motilidad ocular en sentido endotrópico, se descompensó un $25 \%$ y refirió síntomas astenópicos un $28,7 \%$ de ellos.

Palabras clave: Visión binocular, cirugía refractiva, hipermetropía, estereopsis, estrabismo.
$25 \%$ of strabismic patients, and asthenopia symptoms developed in $28.7 \%$ in the same group.

Conclusions: Slight binocular modifications with no symptomatic consequences were found in patients with normal binocular vision. However ocular motility was modified towards esotropia in strabismic patients, binocular decompensation appeared in $25 \%$, and $28.7 \%$ of them suffered from asthenopia (Arch Soc Esp Oftalmol 2006; 81: 107114).

Key words: Binocular vision, refractive surgery, hyperopia, stereopsis, strabismus.

\section{INTRODUCCIÓN}

Una de las complicaciones surgidas tras la cirugía refractiva ha sido la aparición de cuadros de astenopía, diplopía y descompensación de estrabismos. Es una complicación infrecuente hasta la fecha $(1,2)$, aunque no se conoce la incidencia real de estas alteraciones.

En su patogenia pueden intervenir diversos factores, como puede ser la disminución de agudeza visual o la sensibilidad al contraste de uno o ambos ojos, un defecto de refracción residual, anisometropía o aniseiconia inducidas por la cirugía (3-5) o un cambio de ojo dominante, pudiendo resultar estos factores en una modificación temporal o definitiva de la visión binocular.

En un estudio previo de nuestro grupo, se analizó la perturbación de la visión binocular provocada por la cirugía refractiva. Se estudió de forma retrospectiva en pacientes miopes y anisométropes miopes operados con fotoqueratectomía refractiva (PRK). Se encontró una inestabilidad binocular en los primeros meses tras la cirugía, persistiendo una leve disminución de la binocularidad debida principalmente a la disminución de la agudeza visual y la refracción residual (6).

La experiencia en la cirugía de la hipermetropía hasta ahora ha sido menor que en la de la miopía, teniendo entre otras cosas una menor predictibilidad en resultados $(7,8)$. El paciente hipermétrope posee además una mayor labilidad en su visión binocular y se asocia en ocasiones a cuadros de estrabismo. Su binocularidad y grado de compensa- ción oculomotor con frecuencia están ligados a la corrección de su refracción y la cirugía refractiva podría descompensarlos $(9,10)$.

El objetivo de este estudio es analizar, de forma prospectiva, las modificaciones en la visión binocular de hipermétropes y astígmatas hipermetrópicos, tras la cirugía refractiva con técnica LASIK, tanto en pacientes con visión binocular normal como en estrábicos.

\section{SUJETOS, MATERIAL Y MÉTODOS}

Se ha realizado un estudio prospectivo de los pacientes operados entre mayo de 1999 y febrero de 2002, mediante queratomileusis in situ asistida por láser excimer (LASIK) para corregir hipermetropía y astigmatismo hipermetrópico. Todos los pacientes fueron intervenidos por el mismo cirujano (AAP). Para ello se utilizó el láser excimer MEL-70 GScan ${ }^{\circledR}$ (Asclepion-Meditec).

Fueron 31 pacientes (10 hombres y 21 mujeres) de edades comprendidas entre 18 y 59 años, con una edad media de 29,68 DE 8,77 años. Se consideraron en primer lugar aquellos con visión binocular (VB) normal (14), para analizar en segundo lugar los que presentaban alteraciones estrábicas (17).

El protocolo de exploración fue idéntico antes y después de la cirugía, realizándose tras ésta al mes, a los 3 meses, 6 meses, un año y en sucesivas revisiones anuales. El tiempo medio de seguimiento tras la última cirugía fue de 11,10 DE 7,64 meses (3 a 31). El protocolo de exploración incluyó: 
1. Agudeza visual (AV) log MAR con su mejor corrección (Test ETDRS ${ }^{\circledR}$ ). Se consideró «ojo mejor» aquel con mejor agudeza visual corregida, el ojo dominante en los casos en que hubo supresión, el ojo con menor equivalente esférico, y en caso de no existir diferencia, el ojo derecho.

2. Refracción bajo cicloplejía, de la que se anotó su equivalente esférico (EE).

3. Estudio binocular, que incluyó: correspondencia retiniana (sinoptóforo Oculus ${ }^{\circledR}$, tarjetas foveolares), supresión en visión lejana (test vectográfico), estereopsis (test $\mathrm{TNO}^{\circledR}$ de Laméris), amplitud de fusión (sinoptóforo Oculus, tarjetas foveolares), ángulo objetivo en sinoptóforo, correspondiente al grado de foria en pacientes normales y a la desviación en estrábicos. En estos últimos se estudió además el tipo de estrabismo.

Desde el punto de vista motor, 14 pacientes presentaban ortotropia y 17 estrabismo; de ellos, tres fueron endotropias congénitas, nueve esenciales, tres acomodativas y dos exotropias consecutivas. Sensorialmente, los pacientes con ortotropía mostraron correspondencia retiniana normal (CRN); 14 de los 17 pacientes estrábicos presentaron correspondencia retiniana anómala (CRA), y otros tres endotropia acomodativa y CRN. Por ello, desde un punto de vista práctico, se dividió la serie en tres subgrupos: se analizaron en primer lugar aquellos con ortotropia y CRN, en segundo lugar los estrábicos con CRA y finalmente se describió la evolución del grupo de pacientes con endotropía acomodativa y CRN.

Se encontraron ciertas diferencias previas a la cirugía entre los dos grupos principales de la serie. El grupo de estrábicos fue más hipermétrope que el grupo no estrábico (ojo mejor $\mathrm{p}=0,056$, ojo peor $\mathrm{p}=0,019)$. El grado de anisometropía y anisoagudeza también fue mayor en los estrábicos, no siendo en este caso significativo (tabla I).

Tabla I. Descripción de las características refractivas y binoculares de la serie antes y después de la cirugía refractiva dividido por grupos sensoriales

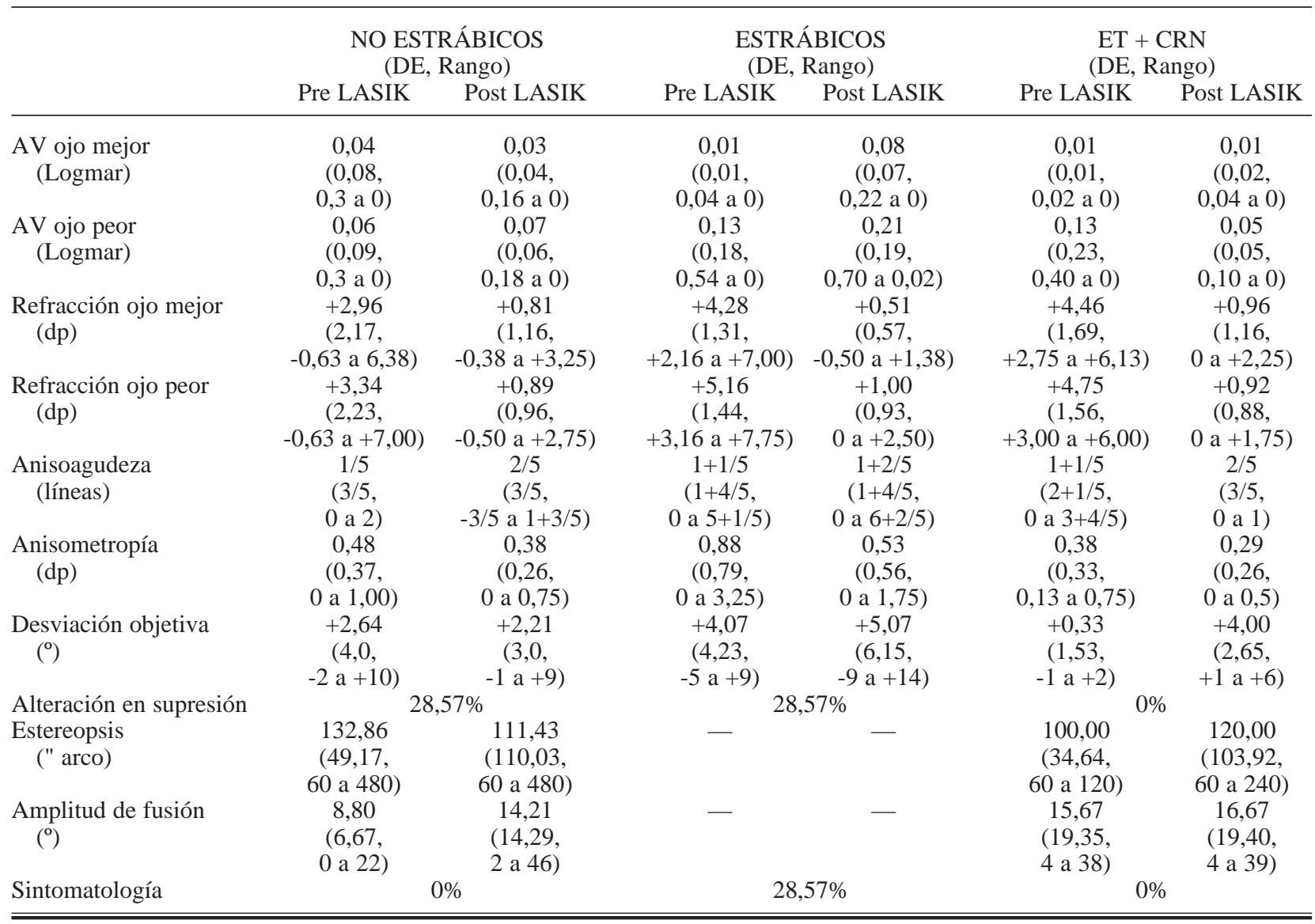

VB: visión binocular, ET: endotropía, DS: desviación estándar, AV: agudeza visual, CRN: correspondencia retiniana normal. 
En la tabla I se puede observar el grado de desviación motora antes de la cirugía. A la hora de evaluar la estabilidad motora, se consideró descompensación significativa cuando la modificación fue mayor o igual a $4^{\circ}$. Desde el punto de vista sensorial, se encontró que los pacientes aparentemente normales presentaron una supresión leve en el $42,86 \%$ de los casos, mientras que todos los estrábicos presentaron supresión intensa, bien de forma monocular $(71,4 \%)$ o bien de forma alternante $(28,6 \%)$.

Para el tratamiento estadístico de datos se utilizó el programa SPSS 11.0 para Windows (SPSS Inc, Chicago, Illinois, EEUU). Se aplicó el test t de Student para muestras independientes y muestras relacionadas, la prueba exacta de Fisher y la correlación de Pearson, con análisis bifactorial. Se consideró que las diferencias eran significativas cuando el $\mathrm{p}$ valor fue menor de 0,05 .

\section{RESULTADOS}

En la tabla I se puede apreciar la situación de los pacientes tras la cirugía refractiva en relación con la agudeza visual, la refracción y la exploración binocular. A la hora de analizar los resultados, se evaluaron las modificaciones sensoriomotoras, observando si habían existido variaciones entre los pacientes con visión binocular normal y los estrábicos.

Se encontró una reducción de la AGUDEZA VISUAL media con respecto a la situación inicial. Esta reducción estuvo en relación con el grado de hipermetropía inicial $(r=-0,276, p=0,03)$. Dado que los pacientes estrábicos presentaron una mayor hipermetropía previa, hallamos en ellos una disminución significativa de la AV, tanto en el ojo mejor $(p=0,001)$, como en el ojo peor $(p=0,015)$, no así en los pacientes normales.

Tras la cirugía se produjo con frecuencia una hipocorrección de la hipermetropía. La refracción final se situó en un rango de $\pm 0,50$ dp en 14 de los 31 pacientes; en otros 12 fue de $+0,50 \mathrm{a}+1,50 \mathrm{dp}$, y en cinco de ellos de $+1,50$ a $+3,00$ dp. Dicha tendencia a la hipermetropía tras la cirugía ocurrió de forma similar en los dos grupos de pacientes.

20 de los 31 pacientes presentaron anisometropía previa a la cirugía $(\geq 0,50 \mathrm{dp})$. En la mayoría de los casos $(90 \%)$ disminuyó, y en el resto se mantuvo estable (media 0,94 DE 0,60 a 0,41 DE 0,44, $\mathrm{p}=0,001$ ).
El grupo no anisométrope $(<0,50 \mathrm{dp})$ fue de 11 pacientes. La cirugía indujo una anisometropía mayor de 0,50 dp en un 36,36\% de ellos, mientras que en el resto se mantuvo estable. El valor medio aumentó desde 0,14 (DE 0,13) hasta 0,49 (DE $0,46)$, no siendo el aumento significativo.

Las modificaciones en la anisometropía no tuvieron repercusión sintomática ni sensorial en ninguno de los grupos.

Por otra parte, siete de los 31 pacientes presentaron anisoagudeza previa mayor o igual a una línea. Esta diferencia de AV mejoró en un 71,43\% y empeoró en un $28,70 \%$ de los casos, pasando el valor medio de 2,97 líneas (DE 1,62) a 2,51 líneas (DE 2,00). Dicha mejoría sin embargo no fue significativa.

Además, la cirugía indujo anisoagudeza en un $20,83 \%$ de los 24 pacientes que no la presentaban previamente, variando la media de $0,09(\mathrm{DE} 0,19)$ a $0,33(\mathrm{DE} 0,42)$ líneas de diferencia $(\mathrm{p}=0,006)$.

Los cambios en la anisoagudeza ocurrieron de forma similar en los pacientes normales y estrábicos, y no tuvieron relación con el resto de parámetros. Sólo un paciente presentó mejor AV en el ojo inicialmente peor; comenzó a suprimir el ojo antes dominante, no mostrando más variaciones ni sintomatología. En el resto de pacientes $(87,5 \%)$, el aumento de anisoagudeza se debió a una pérdida de $\mathrm{AV}$ del ojo peor con respecto al mejor, sin que ello repercutiera de manera subjetiva.

En un importante número de pacientes se modificó la dominancia ocular o fusión de lejos $(25,81 \%)$, con incidencia similar en sujetos normales y estrábicos. Los cambios fueron de distinto tipo: se encontraron pacientes que comenzaron a suprimir un ojo tras la cirugía (3) y otros en que varió en la forma de supresión (4). Entre éstos ocurrió un cambio de dominancia sensorial, en el que pasó a ser dominante el ojo que antes de la cirugía suprimía, alterando su binocularidad. Por último, otro de los pacientes dejó de suprimir (fig. 1).

Al contrario de lo que cabría esperar, en nuestra serie, no se ha podido correlacionar hasta la fecha el cambio en la dominancia con las variaciones de anisometropía o anisoagudeza, ni con repercusiones sintomáticas.

La fusión en sinoptóforo se mantuvo estable en los pacientes normales. Aunque existieron modificaciones clínicas en el grado de amplitud de fusión, no se encontraron diferencias significativas (fig. 2). No se halló ningún paciente estrábico que presentara fusión ni amplitud de fusión tras la cirugía. 


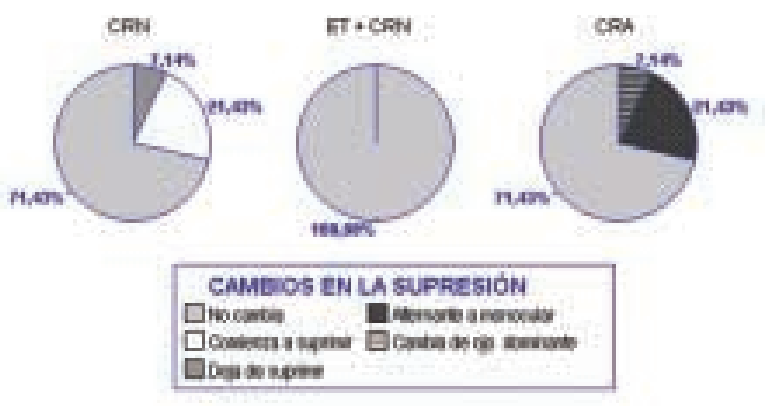

Fig. 1: Alteraciones de la supresión o de la dominancia en los diferentes grupos sensoriales.

No se encontraron modificaciones en relación con la presencia o no de estereopsis, pero sí en cuanto a su intensidad. La estereopsis mejoró en dos de los 14 pacientes normales y empeoró en tres, no siendo las diferencias estadísticamente significativas (fig. 3).

En cuanto a la estabilidad de la motilidad ocular, no se halló ningún caso de estrabismo o diplopía en el grupo de pacientes normales. Existieron modificaciones fóricas en 10 de los 14 pacientes, de pequeña magnitud y de dirección variable, no significativas (fig. 4).

Sin embargo, la desviación ocular en los pacientes estrábicos aumentó, tanto en los pacientes con endotropia ( $\mathrm{p}=0,003)$, como en las exotropias, no siendo en éstas posible el análisis estadístico por el reducido número de sujetos (fig. 5).

De esta forma, se puede considerar que sufrió una descompensación leve $\left(\leq 3^{\circ}\right)$ un $57,14 \%$ de los pacientes estrábicos (todos endotropias, de $+5,25^{\circ}$ DE 2,71 a $+7,00^{\circ}$ DE 2,83$)$. Ocurrió descompensa-

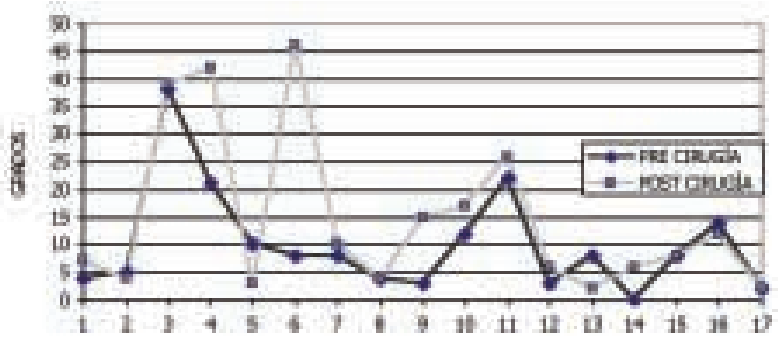

Fig. 2: Grado de amplitud de fusión antes y después de la cirugía. Los pacientes 1 a 3 corresponden a aquéllos con ET acomodativa, los pacientes 4 a 17 a aquellos que presentaron visión binocular normal. La variación media no fue significativa.

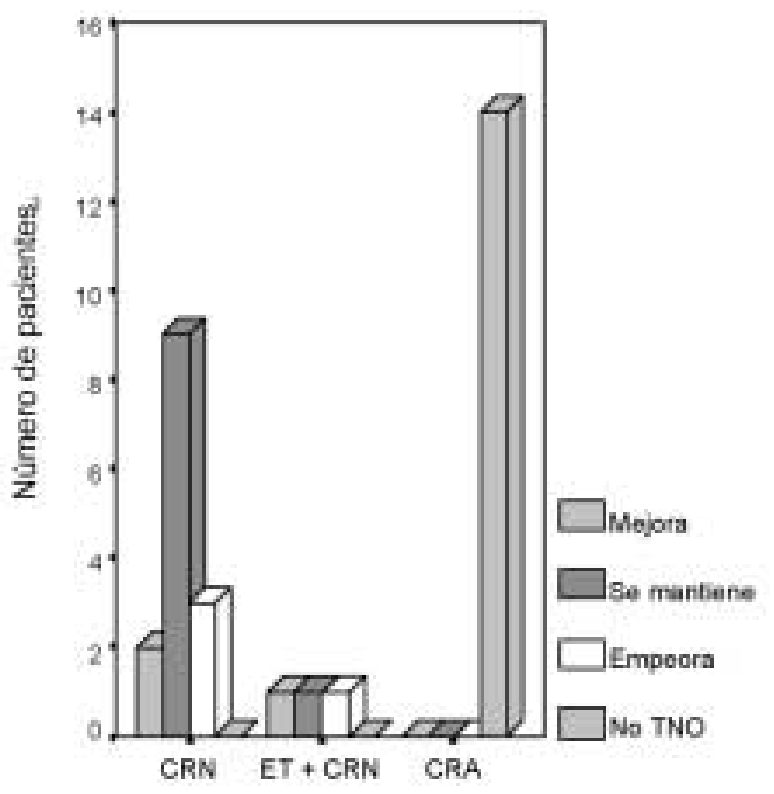

Fig. 3: Cambios ocurridos en la estereopsis, medida con el test TNO, en los tres grupos sensoriales.

ción más intensa $\left(\geq 4^{\circ}\right)$ en un $28,57 \%$ (las endotropías de $+5,50^{\circ} \mathrm{DE} 4,95 \mathrm{a}+10,00^{\circ} \mathrm{DE} 5,66$; las exotropías de $-3,00^{\circ}$ DE 2,83 a $-7,00^{\circ}$ DE 2,83 ) (tabla I).

Un grupo de pacientes $(12,90 \%$ del total) se quejó de síntomas binoculares tras la cirugía refractiva. Las molestias fueron cefaleas, mareos, molestias oculares al fijar la mirada y diplopía por mayor desviación tras esfuerzo visual. Esta sintomatología sólo se presentó en el grupo de pacientes con estrabismo previo, lo que constituye una incidencia de síntomas del 28,57\% de los pacientes estrábicos,

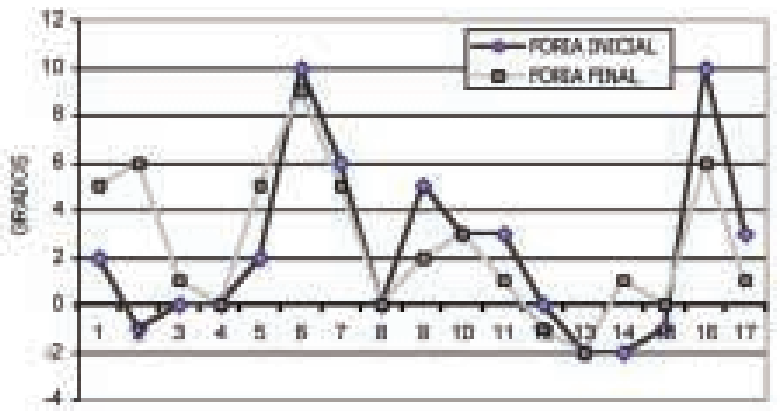

Fig. 4: Variación del grado de foria que presentaron los pacientes con CRN. Los números 1 a 3 corresponden a los pacientes con ET acomodativa y los números 4 a 17 a aquellos con visión binocular normal. La variación media del grado fórico tras la cirugía no fue significativa. 


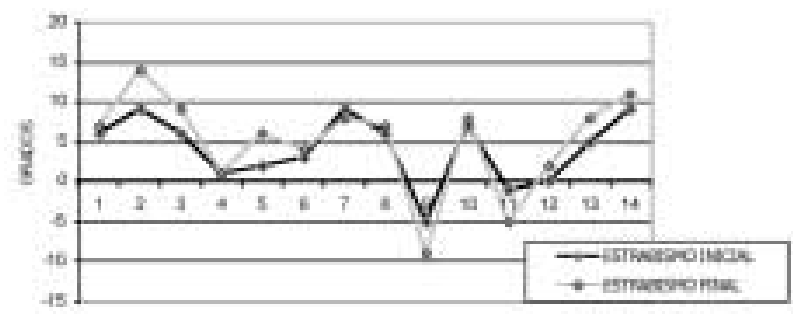

Fig. 5: Modificación del ángulo de desviación de los pacientes estrábicos. Puede observarse la tendencia al aumento de desviación, tanto en los pacientes con endotropia, como en los que presentaron exotropia antes de la cirugía.

frente a incidencia nula en los pacientes normales. Este hecho, sin embargo, no fue estadísticamente significativo (Fisher=0,098).

Finalmente, cabe destacar que el grupo de pacientes con ET acomodativa y CRN (3), quedaron compensados desde el punto de vista motor tras la intervención, persistiendo una leve endoforia no significativa. Tampoco hallamos modificaciones sensoriales en estos pacientes.

\section{DISCUSIÓN}

A la hora de analizar los resultados de nuestra serie, hemos observado que se han producido alteraciones de la sensorialidad tanto en pacientes no estrábicos como estrábicos y de la motilidad ocular fundamentalmente en los pacientes estrábicos.

Aunque en la mayoría de los pacientes los resultados de la cirugía refractiva en cuanto a agudeza visual, refracción, diferencia de AV y grado de anisometropía son bastante satisfactorios, se ha observado que en un porcentaje no despreciable de pacientes estos patrones quedan alterados y por lo tanto pueden influir en la situación sensoriomotora.

\section{Grupo de pacientes no estrábicos}

Los sujetos hipermétropes, aun carentes de alteraciones estrábicas, no poseen una visión binocular perfecta. Antes de la cirugía refractiva, un $42,86 \%$ de los pacientes presentaban supresión leve de un ojo y en un $50 \%$ de ellos, aunque tuvieron estereopsis, fue inferior a 60" de arco.

Tras la intervención se encontraron pequeñas modificaciones en todos los parámetros binoculares explorados. El más significativo fue el cambio en la supresión en visión lejana o dominancia sensorial. Las modificaciones en general fueron leves y ninguno de los pacientes presentó sintomatología binocular como consecuencia de la cirugía.

El origen del cambio en la supresión en este estudio pude ser relacionada con cambios en la $\mathrm{AV}$ o en el grado de anisometropía (en 4 de 14 pacientes), aunque no en todos los casos dichos factores generaran alteraciones de la supresión. La aparición o aumento de anisometropía puede favorecer el desarrollo de una supresión, y al contrario, una mejor fusión en los casos en que disminuya. La repercusión, sin embargo, fue muy individual y no proporcional a la magnitud del cambio. Tampoco se ha observado que la evolución tras la cirugía dependiera de la supresión inicial ni del grado de estereopsis previo de estos pacientes.

De todas formas, creemos que el origen es múltiple y que hay otros factores como la sensibilidad al contraste o la aniseiconia, que también pueden influir en el resultado.

Por lo tanto, las modificaciones en los pacientes no estrábicos fueron leves y ninguno de ellos encontró disconfort ni manifestó insatisfacción tras la intervención. Creemos que su buena visión binocular proporcionó suficiente estabilidad frente a la hipermetropía residual, a las modificaciones de AV o los cambios en la dominancia sensorial.

\section{Grupo de pacientes estrábicos}

Como es frecuente en el grupo de los pacientes estrábicos se encontró previamente a la cirugía refractiva, CRA y supresión en el ángulo subjetivo en el sinoptóforo; también en la evaluación de la fusión de lejos mediante el test vectográfico presentaron todos supresión intensa de un ojo, bien de forma monocular o bien de forma alternante. siete de los 14 pacientes tenían asociado al estrabismo un fuerte componente acomodativo.

Tras la cirugía se encontró una disminución leve de la $\mathrm{AV}$ en ambos ojos, mayor que en los pacientes normales. Esto es debido probablemente a que presentaban una mayor hipermetropía inicial y la cirugía fue más agresiva en éstos. La disminución de AV, de forma mono o binocular, supone la pérdida de calidad visual y puede conducir a una unión binocular más débil. A pesar de ello, en nuestra muestra no hallamos correlación entre dicha disminución y la 
alteración de otros parámetros (como la supresión, el grado de desviación o la sintomatología).

Se encontraron frecuentes modificaciones en la desviación motora, y generalmente en sentido endotrópico. En 10 de los 12 pacientes con endotropia, la desviación aumentó, desde $+5,30^{\circ}$ (DE $2,91) \mathrm{a}+7,60^{\circ}(\mathrm{DE} 3,37)(\mathrm{p}=0,003)$. En las exotropías consecutivas también aumentó, de $-3,00^{\circ}$ (DE 2,83 ) a $-7,00^{\circ}$ (DE 2,83). Dado que se consideró descompensación motora al aumento de $4^{\circ}$ o más de desviación, se encontró una descompensación del estrabismo en un $28,57 \%$ de los pacientes.

Este aumento de la endotropía consideramos que es multifactorial. Destacamos entre dichos factores la hipermetropía residual, que puede influir de manera variable en cada paciente, y que en nuestra serie no se correlacionó de manera significativa. Otro factor a tener en cuenta es el grado de unión binocular, que pueda mantener la estabilidad motora frente a distintos resultados refractivos.

El aumento de desviación motora en un estrábico, además de la repercusión estética, tiene repercusiones sensoriales, tales como levantamientos de supresiones, transitorias o permanentes. Un $28,57 \%$ de los estrábicos se quejó de disconfort en determinadas tareas, fundamentalmente en visión próxima, y diplopía de forma ocasional (un paciente). En nuestra serie no hemos podido relacionarlo con un mayor aumento del ángulo de desviación ni cambios en la supresión, como parecía probable. El único factor que ha afectado de manera clínica, pero no significativa (Fisher 0,098) es el hecho de ser estrábico.

Desde el punto de vista sensorial se encontraron modificaciones en el tipo de supresión en cuatro de los 14 pacientes $(28,57 \%)$; en tres de ellos pasó de alternante a monocular y el otro cambió la dominancia. Este paciente tuvo disconfort acusado tras la cirugía, con descompensación de la exotropía en situaciones de estrés y cansancio.

Por lo tanto, la cirugía refractiva en los pacientes estrábicos, descompensó en un porcentaje no despreciable de casos el equilibrio motor, ya de por sí inestable, provocando un aumento del grado de desviación y la aparición de sintomatología tras la cirugía.

\section{Grupo de pacientes con endotropía acomodativa y CRN}

Tuvimos un pequeño grupo de pacientes con endotropía acomodativa y CRN. Describiremos los resultados obtenidos en estos pacientes, aunque su valor en el análisis estadístico no sea apreciable dado el bajo número de sujetos. Es de esperar que con un mayor número de pacientes se complete este estudio.

En ellos también existieron modificaciones tras la cirugía en algunos de los parámetros. La primera modificación fundamental fue que se compensó la tropía en los tres pacientes a pesar de presentar una refracción residual media de $+0,94$ dp (DE 1,01). Sin embargo, pasaron de una endoforia de $+0,33^{\circ}$ (DE 1,53) a $+4,00^{\circ}$ (DE 2,65). Existió una fuerte correlación en estos casos entre la hipermetropía residual y el grado de endoforia $(\mathrm{r}=0,73)$, sin ser significativa dado el bajo numero de pacientes.

El resto de los parámetros binoculares (supresión, amplitud de fusión y estereopsis), se mantuvieron estables tras la cirugía y ninguno de los tres pacientes tuvo síntomas astenópicos como consecuencia de la intervención.

Por lo tanto aunque es un subgrupo muy pequeño, se encontraron leves modificaciones motoras que sensorialmente no han tenido repercusión.

\section{BIBLIOGRAFÍA}

1. Kushner B, Kowal L. Diplopia after refractive surgery: ocurrence and prevention. Arch Ophthalmol 2003; 121: 315-321.

2. Nemet $P$, Levenger $S$, Nemet A. Refractive surgery for refractive errors which cause strabismus. A report of 8 cases. Binocul Vis Strabismus Q 2002; 17: 187-191.

3. Brooks SE, Johnson D, Fischer N. Anisometropia and binocularity. Ophthalmology 1996; 103: 1139-1143.

4. Tomac S, Birdal E. Effects of anisometropía on binocularity. J Pediatr Ophthalmol Strabismus 2001; 38: 27-33.

5. Antona B. Implicaciones de la Cirugía Refractiva en la Visión Binocular. Arch Optom 2002; 6: 7-22.

6. Gómez de Liaño Sánchez R, Arias Puente A, Ragai Kamel N, Gómez Moreno ML, Gómez de Liaño Sánchez P, Rodríguez JM. Visión binocular tras la cirugía fotorrefractiva. Acta Estrabológica 1996; 25: 185-188.

7. Nagy ZZ, Munkacsy G, Popper M. Photorefractive keratectomy using the meditec MEL $70 \mathrm{G}$-scan laser for hyperopia and hyperopic astigmatism. J Refract Surg 2002; 18: $542-550$.

8. Cobo-Soriano R, Llovet F, Gonzalez-Lopez F, Domingo B, Gomez-Sanz F, Baviera J. Factors that influence outcomes of hyperopic laser in situ keratomileusis. J Cataract Refract Surg 2002; 28: 1530-1538.

9. Hoyos JE, Cigales M, Hoyos-Chacon J, Ferrer J, Maldonado-Bas A. Hyperopic laser in situ keratomileusis for refractive accommodative esotropia. J Cataract Refract Surg 2002; 28: 1522-1529.

10. Stidham DB, Borissova O, Borissov V, Prager TC. Effect of hyperopic laser in situ keratomileusis on ocular alignment and stereopsis in patients with accommodative esotropia. Ophthalmology 2002; 109: 1148-1153. 